\title{
Coexistence of Crohn's Disease and Systemic Lupus Erythematosus with Lupus Nephritis : About Two Cases
}

\author{
Mehdi Somaii ${ }^{1 *}$, Fatma Daoud ${ }^{1}$, Imène Rachdii ${ }^{1}$, Hana Zoubeidi ${ }^{1}$, Besma Ben Dhaou ${ }^{1}$ and Fatma Boussema ${ }^{1}$ \\ ${ }^{1}$ Internal medicine department, Habib Thameur Hospital, Tunis El Manar University, Tunisia
}

*Corresponding author: Mehdi Somaï, Internal medicine department, Habib

Thameur Hospital, Tunis El Manar University, Tunis, Tunisia.

Received Date: August 05, 2019

Published Date: August 09, 2019

\begin{abstract}
Introduction : The systemic lupus erythematosus (SLE) is usually associated with other autoimmune and dysimmune diseases. The coexistence of SLE and Crohn's disease (CD), as an Inflammatory bowel disease, in the same patient is extremely rare. The presence of lupus nephritis during this association is even rarer.

Case Report : We report two observations, a 56-year-old woman and a 37-year-old man. The first was followed for a SLE and after 3 years she developed a CD. The second was followed for a CD and after 4 years he developed a SLE. Both patients presented the second disease at the time that the first was in remission. For both patients, the clinical manifestation of the CD was diarrhoea with ileocecal stenosis. Both patients presented with proliferative lupus nephritis. They had positive anti-DNA antibodies.

Conclusion : Admittedly, the association between the SLE and the CD is extremely rare, especially the presence of lupus nephritis, a screening of one of these diseases in presence of the other is thus justified in front of any several extraintestinal manifestation in CD and any gastrointestinal manifestation in a well-controlled SLE.
\end{abstract}

Keywords: Systemic lupus erythematosus; Crohn's disease; Lupus nephritis

Abbreviations: CD: Crohn's disease; IBD: Inflammatory bowel disease; LN: Lupus nephritis; SLE: Systemic lupus erythematosus

\section{Introduction}

Systemic lupus erythematosus (SLE) is a chronic multisystem inflammatory disease, known as a prototype for autoimmune disease $[1,2]$. It is usually associated with other autoimmune and dysimmune diseases. Inflammatory bowel disease (IBD) is one of the specific dysimmune diseases of the intestine with extraintestinal involvements and with environmental factors and genetic predisposition [3]. IBD is a chronic recurrent disease comprised of two major phenotypes, Crohn's disease (CD) and ulcerative colitis [4]. The coexistence of SLE and CD in the same patient is extremely rare $(<0.4 \%)$ [5]. Even rarer is the association between lupus nephritis (LN) and CD, with only two cases reported to date [6,7]. A review study corroborated that SLE was associated with comorbid CD (OR 2.23) [8]. We communicate herein two cases of patients, ones with SLE who was diagnosed CD colitis and the other with CD who was diagnosed SLE.

\section{Case presentation 1}

A 56-year-old woman with a history of SLE since the age of 53 with renal impairment (proteinuria $=1.44 \mathrm{~g} / 24$ hours, serum creatinine $=357 \mu \mathrm{mol} / \mathrm{L}$, kidney biopsy: Class III $+\mathrm{V}$ ), hematologic impairment (lymphocyte count $=960 / \mathrm{mm} 3$; normal range:1500-4000/mm3 ) and positive anti-DNA, anti-Sm and antinuclear (>1/1600) antibodies and Sjögren syndrome since the age of 53, presented, for a year, a non- bloody liquid diarrhea with a frequency of 4-5 stools per day. The colonoscopy concluded a congestive and ulcerative colitis with a stenosis of the ileocecal valve. Pathological examination of colon biopsies concluded that: The surface coating is sometimes normal, sometimes basophilic, of regenerative aspect. The coating of the crypts is hypercrine, with focal activity or even rare cryptic abscesses. The chorion is edematous and congestive and has a moderate inflammatory 
infiltrate, polymorphous abundance, with some lymphoid nodules but no obvious epithelioid granuloma. The crypts are of variable size and modified architecture. CD has been retained, after also eliminating by several explorations the medicinal, infectious causes such as tuberculosis, inflammatory causes and neoplasia. Magnetic resonance imaging did not show any gastrointestinal thickening or lymphadenopathy and found slight edema at the ileocecal valve level, leaving a margin of $4 \mathrm{~mm}$ Figure 1.

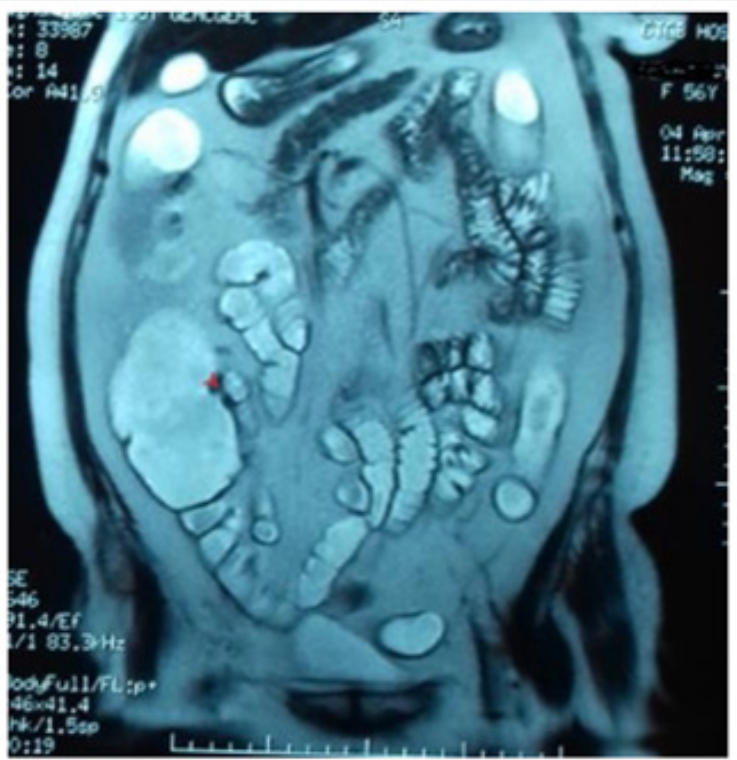

Figure 1: lleocecal stenosis on abdominal magnetic resonance imaging.

The patient was in partial remission for $\mathrm{LN}$ under mycophenolate mofetil. The latter was switched by azathioprine and the corticosteroid dose was increased to $1 \mathrm{mg} / \mathrm{kg} /$ day of prednisone for one month and then gradually decreased. For 12 months, renal remission was maintained and diarrhea disappeared.

\section{Case presentation 2}

A 37-year-old man with a history of stenosing ileocecal Crohn's disease, reported with diarrhea, since the age of 33, for which he had corticosteroid therapy for 2 months and then azathioprine for 12 months. CD has been in remission for 3 years. He had presented skin lesions for one month, including a malar rash. SLE was diagnosed in the presence of malar rash, photosensitivity, proteinuria at $1.24 \mathrm{~g} / 24$ hours, low $\mathrm{C} 3$ (C3 $=0.6 \mathrm{~g} / \mathrm{L}$; normal range: 0.8-1.8 g/L) and C4 (C4=0.1 g/L; normal range: 0.2-0.5 g/L) serum complements, a positive direct Coombs test and positive anti-DNA and anti-nuclear (1/800) antibodies. Renal function was conserved (serum creatinine $=56 \mu \mathrm{mol} / \mathrm{L}$ ). Anatomopathological examination of the renal biopsy concluded LN class III. The patient had 3 pulses of methylprednisolone $1 \mathrm{~g} /$ day then $1 \mathrm{mg} / \mathrm{kg} /$ day of prednisone for one month and progressive decrease. The patient had 6 courses of cyclophosphamide 500mg / 15 days and then a relay of azathioprine. LN has been in complete remission for 9 months.

\section{Discussion}

We described two rare cases of coexistence CD and SLE. The chronology of onset of SLE and CD was different in each of these two observations. Concomitant diagnosis of IBD and SLE is rare and IBD may occur either before or after SLE diagnosis [9]. Although a fortuitous event cannot be ruled out, it is possible that both conditions have certain genetic or immunological abnormalities. However, data on the common genetic susceptibility between SLE and $C D$ are controversial $[10,11]$. In addition, some medications used to treat IBD can cause drug-induced lupus. 5-aminosalicylic acid and sulfasalazine, which are one of the standard treatments for Crohn's disease, constitute a drug-induced lupus-like syndrome. The rare reported cases of lupus-like syndrome induced by 5-aminosalicylic acid and sulfasalazine did not report LN [12-14]. The patient in Presentation 2 never had one of these two drugs. The use of anti-TNF alpha inhibitors in patients with IBD appears to play a role in the formation of anti-nuclear antibodies and / or antidsDNA antibodies. This role is controversial and data on the likely effects of blocking TNF alpha on anti-DNA autoantibody production is always a subject of interest [15]. However, the presence of these antibodies is generally isolated and without clinical symptoms and manifestations of autoimmunity. True cases of SLE in IBD patients treated with TNF alpha inhibitors have been rarely reported $[9,16]$. The patient in Presentation 2 has never had this biotherapy either.

Shor et al. have shown that the prevalence of CD in SLE is highest in the age group between 20 and 59 years [8]. This prevalence was zero at an age less than 20 years. A clear predominance of women has been shown in this review. Nagata et al reported a juvenile case [17]. The delay between the SLE and the CD varied, according to Yamashita's review, between 3 and 36 years. Our two cases were in this interval. To the best of our knowledge, two cases of association between LN and CD have been reported [6,7], and one of them showed favorable renal response to Infliximab [6]. The SLE of one of these two patients preceded the CD [6] while the SLE of the other patient followed the CD [7]. Jit was jst like the case of our two observations. The LN class would be proliferative, because our two patients had a class III and that case of Principi was class IV [6]. We do not have enough detail of Sugimoto's observation because it was written in Japanese [7].

A CD flare can reveal a SLE or be a trigger factor for lupus flare. Nitzan et al. reviewed 10 cases of patients with SLE and IBD [18]. At the moment the second disease was diagnosed the first one was not usually active. It is essential to keep in mind the SLE association with $\mathrm{CD}$, especially if gastrointestinal symptoms develop in a wellcontrolled lupus patient. A colonoscopy with biopsy should be performed in these cases. Likewise, in patients with $C D$ and various extra-intestinal manifestations, the coexistence of SLE should be considered $[19,20]$. Johnson et al. reported a case of CD preceded by SLE with Pyoderma Gangrenosum [21]. In a retrospective review of kidney biopsy specimens obtained from 83 patients with IBD, IgA nephropathy was the most common diagnosis (24\% [20 of 83]), followed by interstitial nephritis (19\% [16 of 83]) [22]. Any secondary glomerular nephropathy in a patient with IBD should suggest an SLE. To the best of our knowledge, all reported cases of SLE associated with CD are positive for anti-DNA antibodies $[18,23]$. De Jager PL et al. shown that the 16q12-13 region, which 
contains the CARD15 Gene variants associated with IBD, is linked to SLE [24]. However, two others studies have infirmed this result $[25,26]$. Further studies are thereby needed to resolve this issue. Our two observations report the rarity of the coexistence of the SLE and the CD. Counting the two cases reported in the literature and our two observations, LN remains extremely rare in CD.

Taking into account the harm that each of these two diseases can cause, it is imperative to screen SLE in a patient with CD having several extraintestinal manifestations and to detect $C D$ in a wellcontrolled SLE with gastrointestinal manifestations.

\section{Conclusion}

Admittedly, the association between the SLE and the CD is extremely rare, especially the presence of lupus nephritis, a screening of one of these diseases in presence of the other is thus justified in front of any several extraintestinal manifestation in CD and any gastrointestinal manifestation in a well-controlled SLE.

\section{Acknowledgement}

None.

\section{Conflict of Interest}

No conflict of interest.

\section{References}

1. Rasaratnam I, Ryan PF (1998) Systemic lupus erythematosus (SLE): changing concepts and challenges for the new millennium. Aust N Z J Med 28(1): 5-11.

2. Cervera R, Khamashta MA, Font J, Sebastiani GD, Gil A, et al. (1993) Systemic lupus erythematosus: clinical and immunologic patterns of disease expression in a cohort of 1000 patients. The European Working Party on Systemic Lupus Erythematosus. Medicine 72(2): 113-124.

3. De Souza HSP (2017) Etiopathogenesis of inflammatory bowel disease: today and tomorrow. Curr Opin Gastroenterol 33(4): 222-229.

4. Silverberg MS, Satsangi J, Ahmad T, Arnott ID, Bernstein CN, et al. (2005) Toward an integrated clinical, molecular and serological classification of inflammatory bowel disease: report of a Working Party of the 2005 Montreal World Congress of Gastroenterology. Can J Gastroenterol Suppl A: 5A-36A.

5. Alves SC, Fasano S, Isenberg DA (2016) Autoimmune gastrointestina complications in patients with systemic lupus erythematosus: case series and literature review. Lupus 25(14): 1509-1519.

6. Principi M, Di Leo A, Ingrosso M, Pisani A, Marangi S, et al. (2004) Lupus nephritis improvement after anti tumor necrosis factor alpha monoclonal antibody (infliximab) treatment for Crohn's disease: A case report. Immunopharmacol Immunotoxicol 26: 243-248.

7. Sugimoto M, Sato Y, Kumagai Y, Suenaga M, Hashimoto H, et al. (1989) A case of systemic lupus erythematosus with lupus nephritis occurring in Crohn's disease. Nihon Naika Gakkai Zasshi 78: 583-584.

8. Shor DB, Dahan S, Comaneshter D, Cohen AD, Amital H, et al. (2016) Does inflammatory bowel disease coexist with systemic lupus erythematosus. Autoimmun Rev 15(11): 1034-1037.
9. Katsanos KH, Voulgari PV, Tsianos EV (2012) Inflammatory bowel disease and lupus: a systematic review of the literature. J Crohns Colitis 6(7): 735-742.

10. Ferreiros Vidal I, Garcia Meijide J, Carreira P, Barros F, Carracedo A, et al. (2003) The three most common CARD15 mutations associated with Crohn's disease and the chromosome 16 susceptibility locus for systemic lupus erythematosus. Rheumatology 42: 570-574.

11. De Jager PL, Graham R, Farwell L, Sawcer S, Richardson A, et al. (2006) The role of inflammatory bowel disease susceptibility loci in multiple sclerosis and systemic lupus erythematosus. Genes Immun 7: 327-334.

12. Kirkpatrick AW, Bookman AA, Habal F (1999) Lupus-like syndrome caused by 5 -aminosalicylic acid in patients with inflammatory bowel disease. Can J Gastroenterol 13(2): 159-162.

13. Carr-Locke DL (1982) Sulfasalazine-induced lupus syndrome in a patient with Crohn's disease. Am J Gastroenterol 77(9): 614-616.

14. Griffiths ID, Kane SP (1977) Sulphasalazine-induced lupus syndrome in ulcerative colitis. Br Med J 2(6096): 1188-1189.

15. Mageed RA, Isenberg DA (2002) Tumour necrosis factor alpha in systemic lupus erythematosus and anti-DNA autoantibody production. Lupus 11: 850-855.

16. Vermeire S, Noman M, Van Assche G, Baert F, Van Steen K, et al. (2003) Autoimmunity associated with anti-tumor necrosis factor alpha treatment in Crohn's disease: a prospective cohort study. Gastroenterology 125(1): 32-39.

17. Nagata M, Ogawa Y, Hisano S, Ueda K (1989) Crohn's disease in systemic lupus erythematosus: a case report. Eur J Pediatr 148: 525-526.

18. Nitzan O, Elias M, Saliba WR (2006) Systemic lupus erythematosus and inflammatory bowel disease. Eur J Intern Med 17: 313-318.

19. Chebli JM, Gaburri PD, De Souza AF, Dias KV, Cimino KO, et al. (2000) Fatal evolution of systemic lupus erythematosus associated with Crohn's disease. Arq Gastroenterol 37: 224-226.

20. Katsanos KH, Voulgari PV, Goussia A, Oikonomou P, Christodoulou DK, et al. (2013) Coexistence of Crohn's disease in a patient with systemic lupus erythematosus. Rheumatol Int 33: 2145-2148.

21. Johnson DA, Diehl AM, Finkelman FD, Cattau EL (1985) Crohn's disease and systemic lupus erythematosus. Am J Gastroenterol 11: 869-870.

22. Ambruzs JM, Walker PD, Larsen CP (2014) The histopathologic spectrum of kidney biopsies in patients with inflammatory bowel disease. Clin J Am Soc Nephrol 9(2): 265-270.

23. Yamashita H, Ueda Y, Kawaguchi H, Suzuki A, Takahashi Y, et al. (2012) Systemic lupus erythematosus complicated by Crohn's disease: A case report and literature review. BMC Gastroenterol 12: 174.

24. De Jager PL, Graham R, Farwell L, Sawcer S, Richardson A et al. (2006) The role of inflammatory bowel disease susceptibility loci in multiple sclerosis and systemic lupus erythematosus. Genes Immun 7: 327-334.

25. Ferreiros-Vidal I, Garcia-Meijide J, Carreira P, Barros F, Carracedo A, et al. (2003) The three most common CARD15 mutations associated with Crohn's disease and the chromosome 16 susceptibility locus for systemic lupus erythematosus. Rheumatol Oxf Engl 42: 570-574.

26. Chong WP, Ip WK, Lau CS, Chan TM, Padyukov L, et al. (2004) Common NOD2 polymorphisms in Hong Kong Chinese patients with systemic lupus erythematosus. Rheumatol Oxf Engl 43: 104-105. 